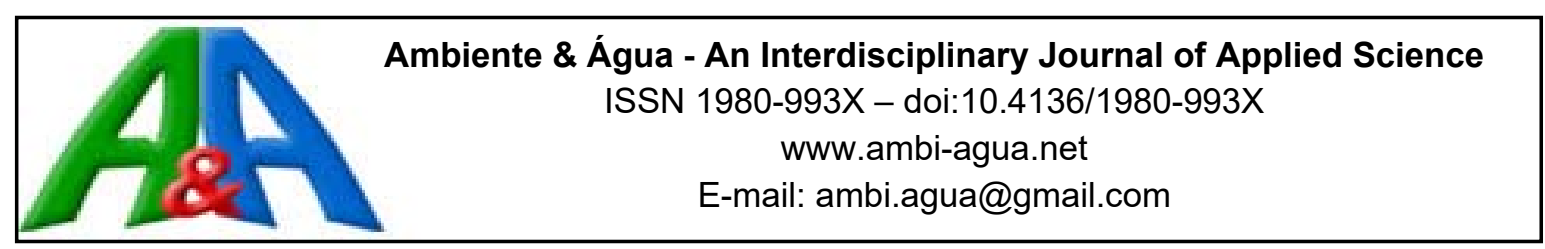

\title{
Emprego dos métodos expeditos de Chow Gumbel e Bell para formulação de equações de chuvas intensas - uma avaliação de desempenho
}

\author{
doi:10.4136/ambi-agua.2001 \\ Received: 09 Sep. 2016; Accepted: 20 Dec. 2016

\begin{abstract}
Karinnie Nascimento de Almeida; José Antonio Tosta dos Reis*; Antônio Sergio Ferreira Mendonça
\end{abstract} \\ Universidade Federal do Espírito Santo (UFES), Vitória, ES, Brasil \\ Departamento de Engenharia Ambiental \\ *Autor correspondente: e-mail: jatreis@gmail.com, \\ kaka.nascimento05@gmail.com, anserfm@terra.com.br
}

\section{RESUMO}

$\mathrm{O}$ adequado dimensionamento de estruturas de micro e macrodrenagem usualmente depende de vazões estimadas com auxílio de equações de chuvas intensas. Séries históricas de precipitações longas e representativas, obtidas através de pluviógrafos, permitem a conformação de equações que relacionem intensidade, duração e frequência de precipitações. Entretanto, em muitas regiões brasileiras não existem pluviógrafos em operação, ou as séries históricas de registros pluviográficos disponíveis são substancialmente mais curtas e menos confiáveis do que aquelas estabelecidas por pluviômetros. Neste contexto, recorrentemente são empregados métodos expeditos para obtenção de equações de chuvas intensas a partir de registros pluviométricos. O presente trabalho tem como objetivo avaliar o desempenho dos métodos expeditos de Chow-Gumbel e de Bell, quando da conformação de equações de chuvas intensas a partir de coeficientes de desagregação disponíveis na literatura técnica corrente. $\mathrm{O}$ teste F de variâncias, assumindo-se nível de significância de 95\%, foi empregado para comparar as respostas das equações de chuvas intensas produzidas pelos métodos expeditos com as respostas de equações de chuvas intensas estabelecidas a partir de registros pluviográficos. Como referência para a análise, foram consideradas equações de chuvas intensas disponíveis para o estado do Paraná. Os resultados indicaram que, para períodos de retorno superiores a 10 anos e durações superiores a 30 minutos, as equações de chuvas intensas determinadas pelos métodos expeditos produzem intensidades equivalentes àquelas determinadas por registros pluviográficos.

Palavras-chave: chuvas intensas, drenagem, equações intensidade-duração-frequência.

\section{Use of the expedited methods of Chow-Gumbel and Bell for formulation of intense rain equations - a performance evaluation}

\begin{abstract}
Adequate design of stormwater structures usually depends on peak runoff rates estimated with the aid of rainfall intensity-duration-frequency (i.d.f.) equations. Long and representative
\end{abstract}


historical precipitation series, obtained by pluviographs, are needed for the estimation of i.d.f. equation parameters. However, in many regions there are no pluviographs in operation or continuous rain gauge records obtained by using this kind of equipment are much shorter and less reliable than those obtained by using manual rain gauge equipment. In this context, expeditious methods are needed for obtaining intensity-duration-frequency equations from daily precipitation records. This study evaluates the performance of the Chow-Gumbel and Bell methods for the establishment of i.d.f. rainfall equations with usual conversion coefficients. The $\mathrm{F}$ test of variance, assuming a 95\% significance level, was used to compare the empirical methods' results with those obtained from pluviographic records. Available pluviographic and regular pluviometers data for stations located in Paraná, state in Brazil were considered. The results are equivalent for return periods greater than 10 years and for durations greater than 30 minutes.

Keywords: extreme rainfall, intensity-duration-frequency equations, urban drainage.

\section{INTRODUÇÃO}

O dimensionamento das diferentes estruturas que conformam os sistemas de micro e macrodrenagem (galerias de águas pluviais, bueiros, sarjetas e reservatórios de detenção em áreas urbanas, por exemplo) é usualmente conduzido a partir de vazões de projeto estimadas por meio de modelos chuva-vazão. O emprego de modelos chuva-vazão, por sua vez, depende de uma equação que relacione intensidade, duração e frequência de precipitações. Neste contexto, a determinação das equações de chuvas intensas constitui, portanto, aspecto central para os projetos de sistemas urbanos de drenagem pluvial (Fendrich, 1999).

Existindo uma rede confiável de pluviógrafos, operada durante período de tempo suficientemente longo e representativo, é possível a definição de relações entre intensidades, durações e frequências de precipitações. Constituem exemplos de equações de chuvas intensas desenvolvidas a partir de registros pluviográficos aquelas apresentadas por Reich (1963), Chen (1983), Kothyari e Garde (1992), Alila (2000), Silva et al. (2000), Falaguasta e Genovez (2003), Oliveira et al. (2005), Hadadin (2005), Back (2006), Singh e Zhang (2007), Garcia et al. (2011) e Fendrich (2011).

Para as situações nas quais não se dispõe de registros pluviográficos, a literatura técnica corrente apresenta diferentes métodos expeditos para a determinação de equações de chuvas intensas, a partir de séries históricas obtidas da operação de pluviômetros. Dentre os métodos expeditos, os métodos de Chow-Gumbel e Bell constituem alternativas que permitem a apropriação de chuvas de diferentes durações e frequências a partir das chuvas máximas anuais de um dia de duração.

No Brasil, em função da limitada disponibilidade de registros pluviográficos, os métodos de Chow Gumbel e Bell são recorrentemente empregados, como nos trabalhos de Almeida et al. (2015), Oliveira et al. (2000), Nali et al. (2007), Rodrigues et al. (2008), Oliveira et al. (2008), Coutinho et al. (2010), Back (2012) e Campos et al. (2014). No entanto, as equações estabelecidas a partir dos referidos métodos usualmente utilizam coeficientes de desagregação de chuvas que não foram localmente determinados. Ainda que diferentes autores (Reich, 1963; Bell, 1969; Genovez e Zuffo, 2000) sugiram que os coeficientes de desagregação se apresentem similares em diferentes partes de mundo, o emprego de coeficientes disponíveis na literatura técnica corrente conduz a intensidades pluviométricas estimadas a partir de equações produzidas sem adequadas calibração e validação.

Neste contexto, o presente trabalho teve como objetivo avaliar o desempenho de equações de chuvas intensas originalmente definidas por Almeida (2015) a partir dos métodos de 
Chow-Gumbel e de Bell, considerados coeficientes de desagregação usualmente empregados no Brasil. Para aplicação do método de Chow-Gumbel foram empregados os coeficientes de desagregação estabelecidos por Pfafstetter (1957) e Occhipinti e Santos (1966). O método de Bell, por sua vez, demandou coeficiente de desagregação sugerido por Righetto (1998). Para avaliação do desempenho das equações conformadas com auxílio dos referidos métodos expeditos foram consideradas, como referência, equações de chuvas intensas desenvolvidas a partir de registros de estações pluviográficas localizados no estado do Paraná.

\section{2. ÁREA DE ESTUDO}

O estado do Paraná está localizado na região sul do Brasil, entre $22^{\circ} 29^{\prime}$ e $26^{\circ} 43^{\prime}$ de latitudes sul e $48^{\circ} 05^{\prime}$ e $54^{\circ} 37^{\prime}$ de longitudes oeste. Segundo a classificação de Köppen, o clima do Paraná é dividido em Af (tropical) sempre úmido, sem estação seca e isento de geadas, na planície litorânea; $\mathrm{Cfa}$ (subtropical) com verões quentes, geadas pouco frequentes e chuvas no verão nas regiões norte, oeste e parte do sudoeste; $\mathrm{Cfb}$ (temperado) com verões frescos, sem estação seca definida, entre o primeiro, segundo e parte do terceiro planaltos (ITCF, 1987).

\section{MATERIAIS E MÉTODOS}

\subsection{Equações de chuvas intensas estabelecidas com auxílio de pluviógrafos.}

As equações de chuvas intensas estabelecidas a partir de registros pluviográficos e disponíveis para o estado que compõe a área de estudo foram estabelecidas por Fendrich (2011). As referidas equações, que constituíram as referências para a análise das respostas oferecidas pelas equações de chuvas intensas conformadas a partir dos métodos expeditos, juntamente com nome e localização das estações pluviográficas e períodos de monitoramento, estão reunidas no Quadro 1. A distribuição espacial das estações pluviográficas no estado do Paraná é apresentada por meio da Figura 1.

Quadro 1. Estações pluviográficas e equações de chuvas intensas disponíveis para o estado do Paraná.

\begin{tabular}{|c|c|c|c|c|c|c|}
\hline Estações & Município & $\begin{array}{l}\text { Altitude } \\
\text { (m) }\end{array}$ & $\begin{array}{c}\text { Latitude S } \\
\text { (Grau Minuto) }\end{array}$ & $\begin{array}{l}\text { Longitude W } \\
\text { (Grau Minuto) }\end{array}$ & Período & Equação \\
\hline Araucária & Araucária & 903 & $25^{\circ} 32^{\prime}$ & $49^{\circ} 23^{\prime}$ & 1989-1999 & $\mathrm{i}=\frac{2505,53 \cdot \mathrm{T}^{0,117}}{(\mathrm{t}+13)^{0,988}}$ \\
\hline Curitiba & Curitiba & 929 & $25^{\circ} 26^{\prime}$ & $49^{\circ} 58^{\prime}$ & $1921-1951$ & $\mathrm{i}=\frac{5950 \cdot \mathrm{T}^{0,217}}{(\mathrm{t}+26)^{1,15}}$ \\
\hline Ivaiporã & Ivaiporã & 650 & $24^{\circ} 15^{\prime}$ & $51^{\circ} 39^{\prime}$ & 1979-1994 & $\mathrm{i}=\frac{676,71 \cdot \mathrm{T}^{0,158}}{(\mathrm{t}+1)^{0,726}}$ \\
\hline Londrina & Londrina & 585 & $23^{\circ} 18^{\prime}$ & $51^{\circ} 09^{\prime}$ & 1975-1985 & $i=\frac{3132,56 \cdot T^{0,093}}{(t+30)^{0,939}}$ \\
\hline Palmital & $\begin{array}{l}\text { Águas } \\
\text { Paraná }\end{array}$ & 840 & $24^{\circ} 53^{\prime}$ & $52^{\circ} 13^{\prime}$ & 1979-1990 & $i=\frac{1548,46 \cdot T^{0,130}}{(t+16)^{0,834}}$ \\
\hline $\begin{array}{c}\text { Porto } \\
\text { Amazonas }\end{array}$ & $\begin{array}{c}\text { Porto } \\
\text { Amazonas }\end{array}$ & 973 & $25^{\circ} 33^{\prime}$ & $49^{\circ} 53^{\prime}$ & 1978-1992 & $\mathrm{i}=\frac{2543,31 \cdot \mathrm{T}^{0,196}}{(\mathrm{t}+27)^{0,952}}$ \\
\hline $\begin{array}{l}\text { Prado } \\
\text { Velho }\end{array}$ & Curitiba & 884 & $25^{\circ} 27^{\prime}$ & $49^{\circ} 15^{\prime}$ & 1981-1999 & $\mathrm{i}=\frac{3221,07 \cdot \mathrm{T}^{0,258}}{(\mathrm{t}+26)^{1,010}}$ \\
\hline Tibagi & Tibagi & 720 & $24^{\circ} 30^{\prime}$ & $50^{\circ} 24^{\prime}$ & 1984-1993 & $\mathrm{i}=\frac{1592,58 \cdot \mathrm{T}^{0,136}}{(\mathrm{t}+11)^{0,882}}$ \\
\hline Tomazina & Tomazina & 483 & $23^{\circ} 46^{\prime}$ & $49^{\circ} 57^{\prime}$ & 1979-1990 & $\mathrm{i}=\frac{2676,7 \cdot \mathrm{T}^{0,149}}{(\mathrm{t}+29)^{0,931}}$ \\
\hline
\end{tabular}

Fonte: Fendrich. (2011). 


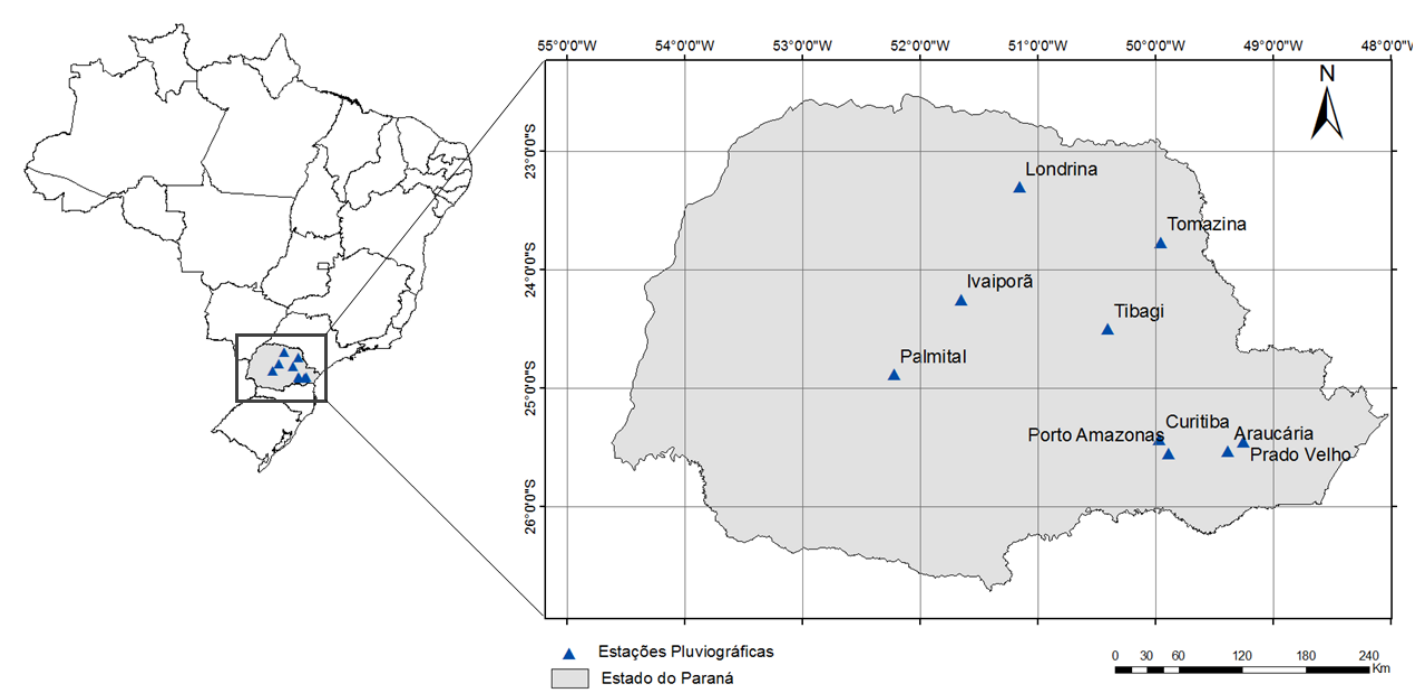

Figura 1. Localização das estações pluviográficas no estado do Paraná.

\subsection{Registros pluviométricos}

Para o estabelecimento de equações de chuvas intensas desenvolvidas a partir dos métodos expeditos de Chow-Gumbel e de Bell foram utilizados registros pluviométricos disponíveis na base de dados gerenciada pela Agência Nacional das Águas (ANA). Foram manipuladas séries históricas consistidas de totais diários de precipitação, registrados em estações pluviométricas instaladas nas mesmas coordenadas geográficas das estações pluviográficas e empregados por Fendrich (2011).

Para a manipulação das séries históricas selecionadas correspondentes aos mesmos períodos analisados no Quadro 1, foi empregado o programa computacional HIDRO, programa de domínio público produzido e disponibilizado pela ANA.

\subsection{Método expedito de Chow-Gumbel}

A aplicação do Método de Chow-Gumbel, apresentado e discutido por CETESB (1986), envolveu as seguintes etapas:

- Seleção das máximas precipitações anuais de 1 dia duração;

- Análise de frequências dos totais precipitados com ajuste da distribuição probabilística de Gumbel à série de máximas precipitações anuais de 1 dia. Dessa forma, foram estimadas as precipitações máximas anuais de 1 dia associadas aos períodos de retorno de 2, 10, 20 e 50 anos;

- Obtenção, a partir dos totais precipitados de 1 dia, das precipitações para durações de 24 horas. Conforme sugerem Occhipinti e Santos (1966), a relação entre as chuvas máximas de 24 horas e 1 dia oscila em torno do valor de 1,14, fator de conversão utilizado no presente trabalho;

É relevante observar que o fator de conversão proposto por Occhipinti e Santos (1966) foi estabelecido a partir da avaliação de precipitações registradas na cidade de São Paulo, não apresentando, portanto, qualquer relação com os registros das estações pluviográficas analisadas por Fendrich (2011).

- Determinação, a partir da avaliação da chuva com duração de 24 horas, das chuvas com mesma frequência de ocorrência, mas com menor duração. Foram empregados, nesta etapa do trabalho, os fatores de conversão apresentados na Tabela 1;

Os fatores de conversão reunidos na Tabela 1 foram estabelecidos por Pfafstetter (1957) a partir da análise de 98 estações pluviográficas instaladas no território brasileiro, incluindo-se 
neste conjunto quatro estações localizadas no estado do Paraná (estações de Curitiba, Jacarezinho, Paranaguá e Ponta Grossa). É relevante observar que apenas uma estação (Curitiba), dentre aquelas analisadas por Pfafstetter (1957), foi também considerada no estudo conduzido por Fendrich (2011). Desta forma, em função dos diferentes conjuntos de estações pluviográficas analisadas, entende-se que os coeficientes propostos por Pfafstetter (1957) não podem ser considerados particulares para o estado do Paraná.

- Estabelecidas as alturas pluviométricas associadas a diferentes períodos de retorno e durações, as intensidades foram produzidas como simples relações entre alturas e durações;

- Definição de equações de chuvas intensas no formato estabelecido pela Equação (1).

$$
\mathrm{i}=\frac{\left(\mathrm{K} \cdot \mathrm{T}^{\mathrm{a}}\right)}{(\mathrm{t}+\mathrm{b})^{\mathrm{c}}}
$$

em que:

i, representa a intensidade máxima ( $\mathrm{mm} /$ minuto),

$\mathrm{T}$, o período de retorno (anos),

$\mathrm{T}$, a duração (minutos) e

$\mathrm{K}$, a, b e c são os parâmetros que localmente se deseja determinar.

O ajuste do modelo estabelecido por meio da Equação 1 foi realizado com auxílio da Programação Não Linear (PNL), aplicada com auxílio do programa Solver, disponível na Planilha Microsoft Excel. Para aplicação da PNL estabeleceu-se uma função objetivo (Equação 2).

$$
\min f_{o}=\sum_{\mathrm{T}} \sum_{\mathrm{t}}\left(\mathrm{i}_{\text {Método }}-\mathrm{i}_{\text {Equação }}\right)
$$

Buscou-se minimizar o erro total entre os valores de intensidade pluviométrica estimados a partir das etapas de 1 a 5 (imétodo) e as intensidades estimadas com auxílio da Equação 1 (iEquação). Os parâmetros $\mathrm{k}, \mathrm{a}, \mathrm{b}$ e c constituíram as variáveis de decisão do problema de otimização, com valores pertencentes ao conjunto $\mathcal{R}+$.

Para a garantia de determinação do ótimo global associado à expressão (02) foram testados diferentes valores iniciais para os parâmetros a, b, c e k. O processo de otimização conduzido com auxílio da PNL foi repetido, no mínimo, 10 (dez) vezes.

Tabela 1. Relação entre as alturas pluviométricas para precipitações de mesma frequência e diferentes durações.

\begin{tabular}{cc}
\hline $\begin{array}{c}\text { Relação entre as alturas } \\
\text { pluviométricas }\end{array}$ & $\begin{array}{c}\text { Coeficientes de } \\
\text { conversão }\end{array}$ \\
\hline $5 \mathrm{~min} / 30 \mathrm{~min}$ & 0,34 \\
$10 \mathrm{~min} / 30 \mathrm{~min}$ & 0,54 \\
$15 \mathrm{~min} / 30 \mathrm{~min}$ & 0,70 \\
$20 \mathrm{~min} / 30 \mathrm{~min}$ & 0,81 \\
$25 \mathrm{~min} / 30 \mathrm{~min}$ & 0,91 \\
$30 \mathrm{~min} / 1 \mathrm{~h}$ & 0,74 \\
$1 \mathrm{~h} / 24 \mathrm{~h}$ & 0,42 \\
$6 \mathrm{~h} / 24 \mathrm{~h}$ & 0,72 \\
\hline
\end{tabular}

Fonte: Pfafstetter (1967). 


\subsection{Método expedito de Bell}

O Método de Bell estima a altura pluviométrica produzida por uma precipitação com duração t e período de retorno $\mathrm{T}$, a partir de uma chuva intensa padrão de 60 minutos de duração e 2 anos de período de retorno (h60,2), conforme Equação 3.

$$
\mathrm{h}_{\mathrm{t}, \mathrm{T}}=\left(a_{0} \cdot \ln \mathrm{T}+a_{1}\right) \cdot\left(a_{2} \cdot \mathrm{t}^{\mathrm{b}}-a_{3}\right) \cdot \mathrm{h}_{60,2}
$$

em que:

a0, $a_{1}, a_{2}, a_{3}$ e b constituem parâmetros regionais.

Conforme Righetto (1998), os referidos parâmetros foram estimados a partir da manipulação de postos pluviográficos instalados em diferentes regiões do País, permitindo a conformação da Equação 4.

$$
h_{t, T}=(0,31 \cdot \ln T+0,70) \cdot\left(0,38 \cdot t^{0,31}-0,39\right) \cdot h_{60,2}
$$

Righetto (1998) sugere, adicionalmente, que o valor de $\mathrm{h}_{60,2}$ pode ser obtido a partir da precipitação máxima diária associada ao período de retorno de 2 anos $\left(\mathrm{h}_{\mathrm{dia}, 2}\right)$, conforme Equação 5.

$$
\mathrm{h}_{60,2} \cong 0,51 . \mathrm{h}_{\mathrm{dia}, 2}
$$

É relevante observar que o fator de conversão $(0,51)$ que conforma a última Equação 5 foi estabelecido a partir da avaliação de precipitações registradas no estado de São Paulo, não apresentando, portanto, qualquer relação com os registros das estações pluviográficas analisadas por Fendrich (2011).

No presente trabalho, a precipitação máxima diária associada ao período de retorno de 2 anos foi apropriada a partir do emprego da distribuição probabilística de Gumbel.

\subsection{Análise estatística}

Para a comparação das respostas oferecidas pelas equações de chuvas intensas determinadas pelos métodos de Chow-Gumbel e de Bell com as equações de chuvas intensas determinadas por Fendrich (2011) foi empregado o teste $\mathrm{F}$ de variância, detalhadamente discutido por Levine et al. (2005). Este procedimento reproduz a abordagem empregada por Almeida et al. (2015), quando da avaliação de respostas de equações de chuvas intensas estabelecidas pelos métodos de Chow-Gumbel e de Bell para o estado da Bahia. A Equação 6 define a estatística do teste $\mathrm{F}$ para a equivalência entre duas variâncias $\left(\mathrm{F}_{\text {est }}\right)$, assumindo-se a independência entre as séries de dados a partir das quais as variâncias foram apropriadas.

$$
\mathrm{F}_{\mathrm{est}}=\frac{\mathrm{S}_{1}^{2}}{\mathrm{~S}_{2}^{2}}
$$

em que:

$S_{1}^{2}$ e $S_{2}^{2}$ representam as variâncias da primeira e segunda amostras, respectivamente.

A primeira amostra é definida como a que possui a maior variância (Levine et al., 2005). Neste estudo, as amostras foram formadas por intensidades de precipitações estimadas pelas diferentes equações de chuvas intensas, para um mesmo valor de duração (t) e período de retorno $(\mathrm{T})$.

Para um determinado nível de significância, a hipótese nula de igualdade entre variâncias de duas amostras é conformada pela Equação 7. A hipótese alternativa de que as variâncias para 
as duas populações não são iguais, por sua vez, é definida pela Equação 8. A hipótese nula será rejeitada se a estatística do teste $\left(\mathrm{F}_{\text {est }}\right)$ for maior do que o valor crítico da distribuição $\mathrm{F}\left(\mathrm{F}_{\text {crítico }}\right)$. Portanto, a regra de decisão é estabelecida pela Equação 9.

$$
\begin{aligned}
& \mathrm{H}_{0}: \mathrm{S}_{1}^{2}=\mathrm{S}_{2}^{2} \\
& \mathrm{H}_{1}: \mathrm{S}_{1}^{2} \neq \mathrm{S}_{2}^{2}
\end{aligned}
$$

$$
\text { Rejeitar } \mathrm{H}_{0} \text { se } \mathrm{F}_{\text {est }}>\mathrm{F}_{\text {crítico }}
$$

A aplicação do teste $\mathrm{F}$ exigiu, adicionalmente, que a probabilidade de $\mathrm{Fest}$ ser menor que o

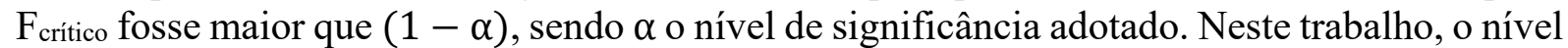
de significância foi de $95 \%$.

A comparação das respostas das equações de chuvas intensas foi realizada para grupos de intensidades pluviométricas associadas aos períodos de retorno de 2, 10, 20 e 50 anos e durações de 5, 30, 60, 240, 720 e 1440 minutos.

\section{RESULTADOS E DISCUSSÃO}

O Quadro 2 reúne as equações de chuvas intensas estabelecidas para o estado do Paraná a partir dos métodos expeditos de Chow-Gumbel e de Bell.

Quadro 2. Equações de chuvas intensas estabelecidas para o estado do Paraná pelos métodos expeditos de Chow-Gumbel e de Bell.

\begin{tabular}{|lll|}
\hline \multirow{2}{*}{ Estação } & \multicolumn{1}{c|}{ Método expedito de determinação das equações IDF } \\
\cline { 2 - 3 } Chow-Gumbel & \multicolumn{1}{c|}{ Bell } \\
\hline Curitiba & $\mathrm{i}=\frac{622,829 \cdot \mathrm{T}^{0,168}}{(\mathrm{t}+2,461)^{1,704}}$ & $\mathrm{~h}_{\mathrm{t}, \mathrm{T}}=(0,31 \cdot \ln \mathrm{T}+0,7) \cdot\left(0,38 \cdot \mathrm{t}^{0,31}-0,39\right) \cdot 37,46$ \\
Ivaiporã & $\mathrm{i}=\frac{569,265 \cdot \mathrm{T}^{0,179}}{(\mathrm{t}+2,444)^{1,704}}$ & $\mathrm{~h}_{\mathrm{t}, \mathrm{T}}=(0,31 \cdot \ln \mathrm{T}+0,7) \cdot\left(0,38 \cdot \mathrm{t}^{0,31}-0,39\right) \cdot 33,88$ \\
Londrina & $\mathrm{i}=\frac{731,645 \cdot \mathrm{T}^{0,188}}{(\mathrm{t}+2,464)^{1,705}}$ & $\mathrm{~h}_{\mathrm{t}, \mathrm{T}}=(0,31 \cdot \ln \mathrm{T}+0,7) \cdot\left(0,38 \cdot \mathrm{t}^{0,31}-0,39\right) \cdot 42,10$ \\
Palmital & $\mathrm{i}=\frac{760,765 \cdot \mathrm{T}^{0,185}}{(\mathrm{t}+2,485)^{1,706}}$ & $\mathrm{~h}_{\mathrm{t}, \mathrm{T}}=(0,31 \cdot \ln \mathrm{T}+0,7) \cdot\left(0,38 \cdot \mathrm{t}^{0,31}-0,39\right) \cdot 43,88$ \\
Porto Amazonas & $\mathrm{i}=\frac{887,594 \cdot \mathrm{T}^{0,135}}{(\mathrm{t}+2,418)^{1,704}}$ & $\mathrm{~h}_{\mathrm{t}, \mathrm{T}}=(0,31 \cdot \ln \mathrm{T}+0,7) \cdot\left(0,38 \cdot \mathrm{t}^{0,31}-0,39\right) \cdot 54,20$ \\
Prado Velho & $\mathrm{i}=\frac{816,563 \cdot \mathrm{T}^{0,182}}{(\mathrm{t}+2,468)^{1,705}}$ & $\mathrm{~h}_{\mathrm{t}, \mathrm{T}}=(0,31 \cdot \ln \mathrm{T}+0,7) \cdot\left(0,38 \cdot \mathrm{t}^{0,31}-0,39\right) \cdot 47,66$ \\
Tibagi & $\mathrm{i}=\frac{657,257 \cdot \mathrm{T}^{0,175}}{(\mathrm{t}+2,481)^{1,705}}$ & $\mathrm{~h}_{\mathrm{t}, \mathrm{T}}=(0,31 \cdot \ln \mathrm{T}+0,7) \cdot\left(0,38 \cdot \mathrm{t}^{0,31}-0,39\right) \cdot 39,00$ \\
Tomazina & $\mathrm{i}=\frac{639,072 \cdot \mathrm{T}^{0,135}}{(\mathrm{t}+2,446)^{1,704}}$ & $\mathrm{~h}_{\mathrm{t}, \mathrm{T}}=(0,31 \cdot \ln \mathrm{T}+0,7) \cdot\left(0,38 \cdot \mathrm{t}^{0,31}-0,39\right) \cdot 38,93$ \\
& $\mathrm{i}=\frac{667,466 \cdot \mathrm{T}^{0,131}}{(\mathrm{t}+2,456)^{1,708}}$ & $\mathrm{~h}_{\mathrm{t}, \mathrm{T}}=(0,31 \cdot \ln \mathrm{T}+0,7) \cdot\left(0,38 \cdot \mathrm{t}^{0,31}-0,39\right) \cdot 40,07$ \\
\hline
\end{tabular}

Para a apropriação das intensidades pluviométricas a partir das equações estabelecidas pelo método de Bell, os valores de altura pluviométrica $(\mathrm{h})$ foram divididos pelos valores de duração (t). Nas equações reunidas no Quadro 2, as intensidades pluviométricas (i) foram obtidas em 
mm.minuto ${ }^{-1}$ a partir de períodos de retorno $(\mathrm{T})$ indicados em anos, alturas pluviométricas em mm e durações em minutos.

Nas Tabelas 2 e 3 estão sumarizados os resultados da aplicação do Teste F de variância para comparação das respostas produzidas pelas equações de Fendrich (2011) e aquelas estabelecidas pelos métodos de Chow-Gumbel e Bell para o estado do Paraná. Nas referidas tabelas, $\mathrm{F}_{\text {est }}$ representa o parâmetro apropriado quando do emprego do teste $\mathrm{F}$ de variância, cujo

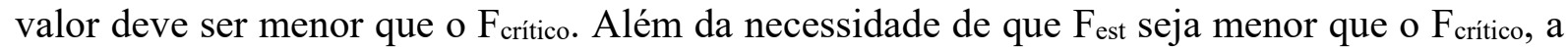
probabilidade de $F_{\text {est }}$ ser menor que o $F_{\text {crítico }}\left(P\left(F_{\text {est }} \leq F_{\text {crítico }}\right)\right)$ deve ser maior que $(1-\alpha)$. Nas tabelas, as células em destaque indicam as combinações de chuvas intensas que se apresentaram equivalentes.

Tabela 2. Resultado da aplicação do teste $\mathrm{F}$ de variância para intensidades pluviométricas associadas aos períodos de retorno de 2 anos e 10 anos.

\begin{tabular}{|c|c|c|c|c|c|c|c|}
\hline \multirow{3}{*}{ Duração (min) } & \multirow{3}{*}{ Métodos pareados } & \multicolumn{6}{|c|}{ Período de Retorno } \\
\hline & & \multicolumn{3}{|c|}{2 anos } & \multicolumn{3}{|c|}{10 anos } \\
\hline & & $\mathrm{F}_{\text {est }}$ & $\mathrm{F}_{\text {crítico }}$ & $\mathrm{P}\left(\mathrm{F}_{\text {est }} \leq \mathrm{F}_{\text {crítico }}\right)$ & $\mathrm{F}_{\text {est }}$ & $\mathrm{F}_{\text {crítico }}$ & $\mathrm{P}\left(\mathrm{F}_{\text {est }} \leq \mathrm{F}_{\text {crítico }}\right)$ \\
\hline \multirow{3}{*}{5} & Gumbel - Bell & 1,19 & 3,44 & 0,41 & 1,18 & 3,44 & 0,41 \\
\hline & Bell - Fendrich (2011) & 3,80 & 3,44 & 0,04 & 2,67 & 3,44 & 0,09 \\
\hline & Gumbel - Fendrich (2011) & 3,19 & 3,44 & 0,06 & 2,26 & 3,44 & 1,14 \\
\hline \multirow{3}{*}{30} & Gumbel - Bell & 1,17 & 3,44 & 0,41 & 1,16 & 3,44 & 0,42 \\
\hline & Bell - Fendrich (2011) & 3,38 & 3,44 & 0,05 & 3,69 & 3,44 & 0,04 \\
\hline & Gumbel - Fendrich (2011) & 3,96 & 3,44 & 0,03 & 4,28 & 3,44 & 0,03 \\
\hline \multirow{3}{*}{60} & Gumbel - Bell & 1,14 & 3,44 & 0,43 & 1,12 & 3,44 & 0,44 \\
\hline & Bell - Fendrich (2011) & 1,26 & 3,44 & 0,37 & 1,84 & 3,44 & 0,20 \\
\hline & Gumbel - Fendrich (2011) & 1,44 & 3,44 & 0,31 & 2,06 & 3,44 & 0,16 \\
\hline \multirow{3}{*}{240} & Gumbel - Bell & 1,22 & 3,44 & 0,39 & 1,22 & 3,44 & 0,39 \\
\hline & Bell - Fendrich (2011) & 1,13 & 3,44 & 0,43 & 1,55 & 3,44 & 0,27 \\
\hline & Gumbel - Fendrich (2011) & 1,38 & 3,44 & 0,33 & 1,28 & 3,44 & 0,37 \\
\hline \multirow{3}{*}{720} & Gumbel - Bell & 1,18 & 3,44 & 0,41 & 1,51 & 3,44 & 0,29 \\
\hline & Bell - Fendrich (2011) & 1,27 & 3,44 & 0,37 & 1,64 & 3,44 & 0,25 \\
\hline & Gumbel - Fendrich (2011) & 1,50 & 3,44 & 0,29 & 1,09 & 3,44 & 0,45 \\
\hline \multirow{3}{*}{1440} & Gumbel - Bell & 2,38 & 3,44 & 0,12 & 1,83 & 3,44 & 0,21 \\
\hline & Bell - Fendrich (2011) & 1,45 & 3,44 & 0,31 & 1,37 & 3,44 & 0,33 \\
\hline & Gumbel - Fendrich (2011) & 3,46 & 3,44 & 0,05 & 1,33 & 3,44 & 0,35 \\
\hline
\end{tabular}

A simples inspeção das Tabelas 2 e 3 permite observar que a aplicação do teste $\mathrm{F}$ de variância indicou, para a área de estudo, a) equivalência entre as intensidades pluviométricas estimadas pelos métodos de Chow-Gumbel e Bell, para quaisquer durações ou períodos de retorno considerados e b) equivalência entre intensidades estimadas pelo método de ChowGumbel e aquelas apropriadas com auxílio das equações estabelecidas por Fendrich (2011) para durações superiores a 30 minutos, independentemente do períodos de retorno avaliado. 
Tabela 3. Resultado da aplicação do teste $\mathrm{F}$ de variância para intensidades pluviométricas associadas aos períodos de retorno de 20 anos e 50 anos.

\begin{tabular}{|c|c|c|c|c|c|c|c|}
\hline \multirow{3}{*}{ Duração (min) } & \multirow{3}{*}{ Métodos pareados } & \multicolumn{6}{|c|}{ Período de Retorno } \\
\hline & & \multicolumn{3}{|c|}{20 anos } & \multicolumn{3}{|c|}{50 anos } \\
\hline & & $\mathrm{F}_{\text {est }}$ & $\mathrm{F}_{\text {crítico }}$ & $\mathrm{P}\left(\mathrm{F}_{\text {est }} \leq \mathrm{F}_{\text {crítico }}\right)$ & $\mathrm{F}_{\text {est }}$ & $\mathrm{F}_{\text {crítico }}$ & $\mathrm{P}\left(\mathrm{F}_{\text {est }} \leq \mathrm{F}_{\text {crítico }}\right)$ \\
\hline \multirow{3}{*}{5} & Gumbel - Bell & 1,23 & 3,44 & 0,39 & 1,30 & 3,44 & 0,36 \\
\hline & Bell - Fendrich (2011) & 2,61 & 3,44 & 0,10 & 2,75 & 3,44 & 0,09 \\
\hline & Gumbel - Fendrich (2011) & 2,12 & 3,44 & 0,15 & 2,11 & 3,44 & 0,15 \\
\hline \multirow{3}{*}{30} & Gumbel - Bell & 1,21 & 3,44 & 0,40 & 1,28 & 3,44 & 0,37 \\
\hline & Bell - Fendrich (2011) & 2,40 & 3,44 & 0,12 & 1,44 & 3,44 & 0,31 \\
\hline & Gumbel - Fendrich (2011) & 2,90 & 3,44 & 0,08 & 1,84 & 3,44 & 0,20 \\
\hline \multirow{3}{*}{60} & Gumbel - Bell & 1,17 & 3,44 & 0,41 & 1,24 & 3,44 & 0,39 \\
\hline & Bell - Fendrich (2011) & 1,64 & 3,44 & 0,25 & 1,23 & 3,44 & 0,39 \\
\hline & Gumbel - Fendrich (2011) & 1,92 & 3,44 & 0,19 & 1,52 & 3,44 & 0,28 \\
\hline \multirow{3}{*}{240} & Gumbel - Bell & 1,17 & 3,44 & 0,42 & 1,11 & 3,44 & 0,45 \\
\hline & Bell - Fendrich (2011) & 1,67 & 3,44 & 0,24 & 1,63 & 3,44 & 0,25 \\
\hline & Gumbel - Fendrich (2011) & 1,44 & 3,44 & 0,31 & 1,48 & 3,44 & 0,30 \\
\hline \multirow{3}{*}{720} & Gumbel - Bell & 1,44 & 3,44 & 0,31 & 1,37 & 3,44 & 0,33 \\
\hline & Bell - Fendrich (2011) & 2,04 & 3,44 & 0,17 & 2,00 & 3,44 & 0,17 \\
\hline & Gumbel - Fendrich (2011) & 1,41 & 3,44 & 0,32 & 1,46 & 3,44 & 0,30 \\
\hline \multirow{3}{*}{1440} & Gumbel - Bell & 1,70 & 3,44 & 0,23 & 1,61 & 3,44 & 0,26 \\
\hline & Bell - Fendrich (2011) & 1,65 & 3,44 & 0,25 & 1,94 & 3,44 & 0,18 \\
\hline & Gumbel - Fendrich (2011) & 1,03 & 3,44 & 0,48 & 1,20 & 3,44 & 0,40 \\
\hline
\end{tabular}

Já a equivalência entre as intensidades pluviométricas estimadas a partir das equações definidas pelos métodos de Bell e por Fendrich (2011) foi observada para todas as durações, considerados os períodos de retorno de 20 e 50 anos. Para o período de retorno de 2 anos, não foi verificada equivalência para a duração de 5 minutos. Para o período de retorno de 10 anos, a equivalência entre intensidades não foi verificada para duração de 30 minutos. É relevante observar, no entanto, que para estes períodos de retorno ( 2 e 10 anos), o valor de $\mathrm{P}\left(\mathrm{F}_{\text {est }} \leq \mathrm{F}_{\text {crítico }}\right)$ assumiu valor substancialmente próximo $\left(\mathrm{P}\left(\mathrm{F}_{\text {est }} \leq \mathrm{F}_{\text {crítico }}\right)=0,04\right)$ do limite estabelecido para garantia de equivalência $(1-\alpha=0,05)$. Neste contexto, eventual flexibilização no nível de significância conduziria à equivalência entre as referidas intensidades.

Assim como observado por Almeida, Reis e Mendonça (2015), quando do estabelecimento de equações de chuvas intensas para o estado da Bahia, as intensidades pluviométricas estimadas pelos métodos de Chow-Gumbel e Bell apresentaram-se equivalentes, ao nível de significância de $95 \%$, para quaisquer durações ou períodos de retorno avaliados. Os referidos autores indicaram, no entanto, que a equivalência entre as equações conformadas com métodos de Chou-Gumbel e de Bell e as equações estabelecidas a partir de registros pluviográfícos foi verificada apenas para períodos de retorno superiores a 10 anos e durações maiores que 30 minutos. 
É relevante observar que, em projetos de drenagem pluvial, a duração da chuva é, usualmente, o tempo de concentração da bacia objeto da atuação do sistema de drenagem. Desta forma, excluindo-se bacias de pequenas dimensões (para as quais os tempos de concentração seriam inferiores a 30 minutos), os métodos expeditos podem constituir alternativa útil para apropriação das chuvas de projeto. Também constitui prática dos projetos de drenagem a adoção de intensidades pluviométricas associadas a períodos de retorno de 20 anos ou mais, frequência de ocorrência de precipitações para a qual os métodos expeditos também se apresentaram como alternativa consistente.

\section{CONCLUSÕES}

O presente estudo teve como principal objetivo avaliar o desempenho de equações de chuvas intensas definidas a partir dos métodos de Chow-Gumbel e Bell, métodos expeditos que envolvem o emprego de fatores de conversão entre chuvas de diferentes durações e que são recorrentemente empregados em avaliações de chuvas intensas em todo o território brasileiro. Como referência para avaliação do desempenho das referidas equações e, portanto, da consistência dos fatores de conversão que envolvem, foram consideradas equações de chuvas intensas desenvolvidas para o estado do Paraná a partir da análise de registros pluviográficos.

As principais conclusões do presente estudo podem ser assim sumarizadas:

- As intensidades pluviométricas estimadas para o estado do Paraná por equações desenvolvidas com o emprego dos métodos de Chow-Gumbel e de Bell apresentaram-se equivalentes ao nível de significância de $95 \%$, independentemente da duração ou período de retorno avaliados;

- As intensidades estimadas por equações definidas com o auxílio dos métodos de ChowGumbel e Bell apresentaram-se equivalentes àquelas estimadas a partir de equações conformadas com registros pluviográficos de chuvas com durações superiores a 30 minutos e períodos de retorno compreendidos entre 2 e 50 anos.

\section{REFERÊNCIAS}

ALILA, Y. Regional rainfall depth duration frequency equations for Canada. Water Resources Research, v. 36, n. 7, p. 1767-1778, 2000. 10.1029/2000WR900046

ALMEIDA, K. N. Avaliação do desempenho dos métodos expeditos de determinação de equações de intensidade-duração-frequência. 2015. 75f. Monografia (Graduação em Engenharia Ambiental) - Departamento de Engenharia Ambiental, Universidade Federal do Espírito Santo, Vitória, 2015.

ALMEIDA, K. N.; REIS, J. A. T.; MENDONÇA, A. S. F. Avaliação do desempenho dos métodos expeditos de determinação de equações de chuvas intensas. Revista Brasileira de Ciências Ambientais, v. 35, p. 63-77, 2015.

BACK, A. J. Relações de intensidade-duração-frequência de chuvas intensas de Chapecó, Estado de Santa Catarina. Revista Acta Scientiarum, v. 28, n. 4, p. 575-581, 2006.

BACK, A. J.; OLIVEIRA, J. L. R.; HENN, A. Relações entre precipitações intensas de diferentes durações para desagregação da chuva diária em Santa Catarina. Revista Brasileira de Engenharia Agrícola e Ambiental, v. 16, n. 4, p. 391-398, 2012. 
BELL, F. C. Generalized rainfall-duration-frequency relationships. Journal of the Hydraulics Division, v. 95, n. HY1, p. 311-327, 1969.

CAMPOS, A.R.; SANTOS, G. G.; SILVA, S.B.C.; FILHO, J. I.; LOURA. D. S. Equações de intensidade-duração-frequência de chuva para o estado do Piauí. Revista Ciência e Agronomia, v. 45, n. 3, p. 488-498, 2014.

CETESB - COMPANHIA DE TECNOLOGIA DE SANEAMENTO AMBIENTAL. Drenagem Urbana - Manual de Projeto. São Paulo: CETESB/ASCETESB, 1986.

CHEN, C. Rainfall intensity-duration-frequency formulas. Journal of Hydraulic Engineering, v. 109, n. 12, p. 1603-1621, 1983. http://dx.doi.org/10.1061/(ASCE)07339429(1983)109:12(1603)

COUTINHO, A. P.; SILVA, F. B.; SILVA, R. O.; ANTONINO, A. C. D.; MONTENEGRO, S. M. G. L. Determinação de equações de chuvas intensas para municípios das mesorregiões do estado de Pernambuco com dados pluviométricos In: SIMPÓSIO DE RECURSOS HÍDRICOS DO NORDESTE, 10., 2010, Fortaleza. Anais... Fortaleza: ABRH, 2010. 1 CD-ROM.

FALAGUASTA, L. N.; GENOVEZ, A. M. Equações de chuvas intensas para os estados de São Paulo e Paraná. Revista Brasileira de Recursos Hídricos, v. 8, n. 3, p. 169-176, 2003. http://unicamp.sibi.usp.br/handle/SBURI/13414

FENDRICH, R. Chuvas intensas para obras de drenagem no estado do Paraná. Curitiba: Gráfica Vicentina Editora, 2011.

FENDRICH, R. Importância das Equações de Chuvas Intensas na Drenagem Urbana. In: SIMPÓSIO BRASILEIRO DE RECURSOS HÍDRICOS, 13., 1999, Belo Horizonte. Anais... Belo Horizonte: ABRH, 1999. 1 CD-ROM.

GARCIA, S. S.; AMORIM, R. S. S.; COUTO, E. G.; STOPA, W. H. Determinação de equações intensidade-duração-frequência para três estações meteorológicas do estado do Mato Grosso. Revista Brasileira de Engenharia Agrícola e Ambiental, v. 15, n. 6, p. 575$581,2011$.

GENOVEZ, A. M.; ZUFFO, A. C. Chuvas intensas no estado de São Paulo. Revista Brasileira de Recursos Hídricos. v .5, n. 3, p. 45-58, 2000.

HADADIN, N. A. Rainfall Intensity-Duration-Frequency Relationship in the Mujib Basin in Jordan. Journal of Applied Sciences, v. 5, p. 1777-1784, 2005. http://dx.doi.org/10.3923/jas.2005.1777.1784

ITCF - INSTITUTO DE TERRAS, CARTOGRAFIA E FLORESTAS. Atlas do Estado do Paraná. Curitiba, 1987.

KOTHYARI, U. C.; GARDE, R. J. Rainfall intensity-duration-frequency formula for India. Journal of Hydraulic Engineering, v. 118, n. 2, p. 323-336, 1992. http://dx.doi.org/10.1061/(ASCE)0733-9429(1992)118:2(323)

LEVINE, D. M.; STEPHAN, D.; KREHBIEL, T. C.; BERENSON, M. L. Estatística - Teoria e Aplicações. Rio de Janeiro: Livros Técnicos e Científicos, 2005. 
NALI, J. O.; REIS, J. A. T.; REIS, A. O. P.; SILVA, F. G. B. Avaliação da resposta de diferentes de equações de chuvas intensas obtidas para as bacias hidrográficas dos rios Jucu e Santa Maria da Vitória, ES. In: SIMPÓSIO BRASILEIRO DE RECURSOS HÍDRICOS, 12. 2007. Anais... São Paulo: ABRH, 2007. 1 CD-ROM.

OCCHIPINTI, A. G.; SANTOS, P. M. Relação entre as precipitações de "Um Dia" e "24 Horas" na cidade de São Paulo. São Paulo: Instituto Astronômico e Geofísico - USP, 1966.

OLIVEIRA, L. F. C.; ANTONINI, J. C. A., GRIEBELER, N. P. Estimativas de chuvas intensas para o estado de Goiás. Revista de Engenharia Agrícola, v. 28, n. 1, p. 22-33, 2008.

OLIVEIRA, L. F. C.; CORTÊS, F. C.; BARBOSA, F. O. A.; ROMÃO, P. A.; CARVALHO D. F. Estimativa das equações de chuvas intensas para algumas localidades no Estado de Goiás pelo método da desagregação de chuvas. Pesquisa Agropecuária Tropical, v. 30, n. 1, p. 23-27, 2000.

OLIVEIRA, L. F. C.; CORTÊS, F. C., WEHR, T. R. Intensidade-Duração-Frequência de chuvas intensas para localidades no estado de Goiás e Distrito Federal. Pesquisa Agropecuária Tropical, v. 35, n. 1, p. 13-18, 2005.

PFAFSTETTER. O. Chuvas Intensas no Brasil. Rio de Janeiro: DNOS, 1957.

REICH, B. M. Short-duration rainfall-intensity estimates and other design aids for regions of sparse data. Journal of Hydrology, v. 1, n. 1, p. 3-28, 1963. http://dx.doi.org/10.1016/0022-1694(63)90029-5

RIGHETTO, A. M. Hidrologia e Recursos Hídricos. São Calos: EESC/USP, 1998.

RODRIGUES, J. O.; ANDRADE, E. M.; OLIVEIRA, T. S.; LOBATO, F. A. O. Equações de intensidade-duração-frequência de chuvas para localidades de fortaleza e pentecoste. Scientia Agraria, v. 9, n. 4, p. 511-519, 2008.

SILVA, D. D.; FILHO, R. R. G.; PRUSKI, F. F.; PEREIRA, S. B.; NOVAES, L. F. Chuvas intensas no estado da Bahia. Revista Brasileira de Engenharia Agrícola e Ambiental, v. 6, n. 2, p. 362-367, 2000.

SINGH, V. P.; ZHANG, L. IDF curves using the Frank Archimedean copula. Journal of Hydrologic Engineering, v. 12, n. 6, p. 651-662, 2007. http://dx.doi.org/10.1061/(ASCE)1084-0699(2007)12:6(651) 